tumour-suppressor gene (VHL), which hobbles the gene's ability to express the tumour suppressing protein. Baylin and Herman showed that $20 \%$ of the remaining patients with the non-inherited form of RCC did not have a mutation in VHL. Their genes were silenced not by a mutation but rather by hypermethylation ${ }^{2}$.

The following year, in collaboration with Sidransky's lab at Johns Hopkins, Baylin and Herman showed that human cancers commonly arise when a particular tumour suppressor gene, known as p16, is hypermethylated. Moreover, in many cancers including RCC, epigenetic and genetic mutations often work in tandem: one of the two copies of a tumour suppressor gene is inactivated by genetic mutation, while the other is hypermethylated. This finding "convinced us that epigenetic abnormalities could play an important driving role in cancer - and we and many others have been pursuing this possibility ever since," says Baylin.

The move from a purely genetic to an epigenetic model is crucial for prevention strategies. As numerous gene therapy trials have shown, it is very difficult to treat a genetic disease by re-activating the dormant, mutated gene or by replacing it with a non-mutated one. "Epigenetic changes are reversible, and therefore have an edge over genetics," says Mukesh Verma, an epigeneticist at the National Cancer Institute's division of cancer control and population sciences in Bethesda, Maryland. Furthermore, epigenetic changes in cancer occur
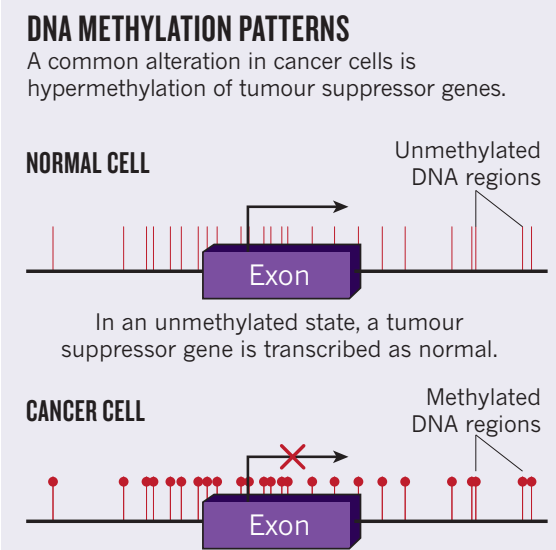

Tumour suppressor gene is hypermethylated and is not transcribed, leading to loss of its normal cellular function.

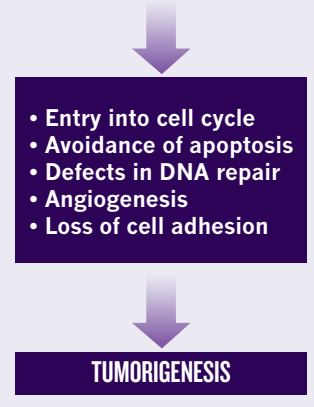

before genetic mutations. "If you can prevent methylation of those tumour suppressor genes, you might have a valuable prevention strategy," says Baylin.

\section{THE ENVIRONMENTAL LINK}

Epigenetics has also provided clues that link environmental factors with cancerous genetic changes. Changes in methylation can be detected in the blood of cancer-free individuals who smoke and eat high-fat diets, and these changes have been shown to precede genetic mutations ${ }^{3}$. More recently, Karl Kelsey, a molecular epidemiologist at Brown University in Providence, Rhode Island, has uncovered independent associations between epigenetic patterns in breast cancer tumours, the tumour size, alcohol consumption and folate intake ${ }^{4}$.

A prime candidate at the interface of environment and genetics is chronic inflammation, which is known to precede the development of numerous types of precancerous lesions - and indeed certain cancers themselves, including oesophageal, liver and colon cancers. Inflammation has been linked with increased DNA methylation in otherwise healthy looking tissue. Issa calls chronic inflammation "a truly epigenetic phenomenon".

Long-term inflammation may result from infection with Helicobacter pylori or hepatitis $\mathrm{C}$ virus, or from autoimmune diseases such as ulcerative colitis (a form of inflammatory bowel disease). People with ulcerative colitis often develop colon cancer at a younger age - for example in their 50s - than the 60 to 70 year average age of onset. "All of the epigenetic changes that occur in colon cancer, specifically DNA hypermethylation and gene silencing, are accelerated - and appear in the inflamed tissue prior to actual cancer," says Baylin.

Half the world's population is infected with inflammation-causing $H$. pylori, yet stomach cancer afflicts barely $0.03 \%$ of those. "There must be something in the body itself that makes it react differently to infection," says Emad El-Omar, a gastroenterologist at the University of Aberdeen in Scotland. El-Omar is investigating whether genetic variation can influence this response

Genetic polymorphisms are normal genetic variations within a population that can subtly raise or lower each person's levels of a particular protein. While everyone within the normal population produces the same pro- and antiinflammatory chemicals (or cytokines) an individual's particular levels vary according to genetic make-up. Certain polymorphisms, El-Omar hypothesized, might tip the balance towards chronic inflammation and then to cancer.

El-Omar found that polymorphisms in the inflammation-related IL-1B and transforming growth factor (TNF)- $\alpha$ genes determine the levels of circulating IL-1B and TNF- $\alpha$ pro-inflammatory cytokines. People with a genotype disposed to higher levels of these cytokines have an increased risk of developing stomach cancer following $H$. pylori infection. El-Omar has made similar discoveries in other cancers. In colorectal cancer, for example, he discovered there is an inflammatory environment in and around premalignant lesions. Within this region he found nine differentially expressed genes linked with inflammation, including those responsible for IL-8 and the CXCL- family of cytokines ${ }^{5}$.

Others researchers are making similar connections. For example, there is evidence that pro-inflammatory prostaglandin endoperoxide synthase ${ }^{6}$ and TGF- $\beta^{7}$ are both significantly associated with increased risk of colon cancer. Although the evidence linking inflammation to epigenetics and cancer is mounting, the underlying mechanism of the association and the value of screening for these polymorphisms remain less clear.

\section{POTENTIAL FOR REVERSAL}

Drugs and dietary substances that alter epigenetic pathways are currently being tested. During his research on RCC, for example, Baylin and colleagues were able to reverse hypermethylation of the VHL gene with the drug 5-azacytidine. Trials of demethylating drugs as adjuvant treatments to prevent lung cancer recurrence are underway. If successful, prevention trials are the next logical step. "We need five- and ten-year survival data with current drugs to be sure there are no secondary effects before we give them to reasonably healthy people for prevention," says Issa. He sees a different source for the first wave of preventive medications. "I would bank on discovering more 'gentle' approaches to epigenetic manipulation for cancer prevention - be they natural products, existing drugs with a good safety records, or even vitamins or diet."

Epigenetic changes also have potential utility as biomarkers (see Portents of malignancy, page S19). Being able to read the methylation pattern of certain genes could give scientists a heads-up for people on the brink of developing cancer.

Slowly the importance of the epigenome in cancer development is being appreciated. "Geneticists are hugely more aware of the importance of epigenetics in the development of cancer," observes Baylin. When it comes to cancer prevention, the future could lie in arresting the reversible epigenetic changes before irreversible mutations take hold.

Vicki Brower is a freelance writer in New York.

1. Jones, S, et al. Science 330, 228-231 (2010).

2. Herman, J.G. et al. PNAS USA 91, 9700-9704, (1994)

3. Brait, M. et al. Cancer Epidemiol Biomarkers Prev. 18, 2984-2991 (2009).

4. Christensen, B. C. et al. PLoS Genetics 6(7):e1001043 (2010).

5. McLean, M. H. et al. PLoS One 6 (1), e15366 (2011).

6. Frank, B. et al. Cancer Genetics 127, 2822-2830 (2010).

7. Slattery, M. et al. Cancer Epidemio.I Biomarkers

Prevention. 20, 57 (2011). 